University of Nebraska - Lincoln

DigitalCommons@University of Nebraska - Lincoln

Faculty Publications, Department of Physics and Astronomy

Research Papers in Physics and Astronomy

1978

\title{
Evolved Contact Systems of Spectral Type A: Au Puppis, V535 Arae, and V1073 CYGNI
}

\author{
Kam-Ching Leung \\ University of Nebraska-Lincoln, kleung2@unl.edu \\ Donald P. Schneider \\ University of Nebraska-Lincoln, dps7@psu.edu
}

Follow this and additional works at: https://digitalcommons.unl.edu/physicsfacpub

Part of the Physics Commons

Leung, Kam-Ching and Schneider, Donald P., "Evolved Contact Systems of Spectral Type A: Au Puppis, V535 Arae, and V1073 CYGNI" (1978). Faculty Publications, Department of Physics and Astronomy. 84. https://digitalcommons.unl.edu/physicsfacpub/84

This Article is brought to you for free and open access by the Research Papers in Physics and Astronomy at DigitalCommons@University of Nebraska - Lincoln. It has been accepted for inclusion in Faculty Publications, Department of Physics and Astronomy by an authorized administrator of DigitalCommons@University of Nebraska Lincoln. 
The Astrophysical Journal, 222:917-923, 1978 June 15

(C) 1978. The American Astronomical Society. All rights reserved. Printed in U.S.A.

\title{
EVOLVED CONTACT SYSTEMS OF SPECTRAL TYPE A: AU PUPPIS, V535 ARAE, AND V1073 CYGNI
}

\author{
KaM-ChING LeUNG \\ Department of Physics and Astronomy, University of Nebraska; and Office of Energy Research, \\ U.S. Department of Energy, Washington
}

AND

DONALD P. SCHNEIDER

Department of Physics and Astronomy, University of Nebraska; and Hale Observatories, California Institute of Technology, Carnegie Institution of Washington

Received 1977 August 17; accepted 1978 January 18

\begin{abstract}
The photoelectric observations of AU Pup, V535 Ara, and V1073 Cyg were reanalyzed with Wilson and Devinney's approach. All three systems are found to be contact configurations. AU Pup has an unusually large degree of overcontact, $72 \%$. V1073 Cyg is found to be an evolved contact system since the radius of the primary is twice that of a zero-age main-sequence star of corresponding mass. All three systems are found to be evolved according to their locations in the period-spectral-type diagram. Improved photometric parameters are reported for all three systems.
\end{abstract}

Subject heading: stars: eclipsing binaries

\section{INTRODUCTION}

The term "contact binary" has been used in the literature rather loosely for some time. Many W UMa type and $\beta$ Lyrae type eclipsing binaries were believed to be contact systems (e.g., Eggen 1961). These findings were usually based on (i) the period and spectral types and/or (ii) the photometric solutions using the conventional approaches of Russell and Merrill (1952) and of Kopal (1959). In recent years more realistic approaches employing the Roche model have been developed. The new approaches handle close systems well. The major contributors in this area of research are Lucy (1968), Hill and Hutchings (1970), Wilson and Devinney (1971), and Mochnacki and Doughty (1972).

As a result of these new approaches, many systems which were believed to be in contact have been proved to be either semidetached or detached systems and vice versa. In this paper, we investigate those systems of spectral type A which are promising candidates for having contact configuration. All of these systems, AU Pup (Chambliss 1968), V535 Ara (Chambliss 1967), and V1073 Cyg (Kondo 1966), have their sums of side radii equal to or larger than 0.75 (with respect to the distance between the centers of components) according to the conventional Russell and Merrill (1952) approach.

\section{GENERAL APPROACH}

In order to investigate whether these systems were truly in contact, we adopted the Wilson and Devinney (1971) approach. To begin with, we always assumed a system to be detached. Normally, we started with the light curve program, and by trial and error a set of parameters was obtained which marginally represented the observations. With this final set of trial parameters, we proceeded with the differential corrections program, starting with detached configuration, mode 2 (see Leung and Wilson 1977 for detailed description of the modes of operation), and let the process evolve to its final solution. The solution usually evolved from a completely detached configuration (mode 2) into semidetached configurations; mode 4 (star $1=$ component eclipsed at the primary minimum, filling its Roche surface) or mode 5 (star 2 filling its Roche surface); then gradually settled into a contact configuration (mode 3 ). A final set of parameters was adopted when the corrections to the adjustable parameters were similar to or smaller than the probable errors.

Normal points were used for the analysis, and they were weighted according to the number of individual observations. The scatter is attributed to shot noise. Both yellow and blue light curves were employed simultaneously in deriving the photometric solution. In all cases, we assumed that the gravity darkening coefficients $g_{1}=g_{2}=1$ (radiative atmospheres) and the bolometric albedos $A_{1}=A_{2}=1$ (full reradiation). The secondary luminosity was calculated in the program from the Planck function, geometry, and the primary luminosity. The adjustable parameters were $i$, the inclination angle; $L_{1}$, the luminosity of the primary (star 1$) ; \Omega_{1}$ and $\Omega_{2}$, the surface potentials; $T_{2}$, the polar temperature of the secondary; and $q$, the mass ratio $\left(m_{2} / m_{1}\right)$. The capability to determine this last parameter, $q$, from the light curve alone has become possible only with the advent of modern models. If the system is completely detached (negligible tidal distortion), however, even these approaches obviously 
cannot distinguish between various $q$ values. The program will simply adjust the surface potentials of the components to get the correct fractional radii for any mass ratio. For these systems classical approaches, such as that of Russell and Merrill (1952), will work just as well.

In theory, as the system approaches a contact configuration, the components become distorted, and only one value for the mass ratio can reproduce the light curve. The effect of different $q$ values, however, is fairly small, and is often masked by uncertainty in the observations. Factors which enhance the possibility of determining the mass ratio from the light curve alone are (1) a large degree of overcontact, which increases the distortion; and (2) a high inclination, which gives deep eclipses. For a system such as $\mathrm{BH}$ Cen (Leung and Schneider 1977), in which both eclipses are over $1 \mathrm{mag}$ ( $i$ of $90^{\circ}$ and overcontact of $20 \%$ ), one can determine the mass ratio with a fair degree of confidence. On the other hand, one cannot distinguish values of the photometric mass ratio for the system AO Cas (marginal contact, and $0.15 \mathrm{mag}$ eclipses) despite excellent observations. Unfortunately, very few systems exhibit BH Cen characteristics, so there is usually some ambiguity in the mass ratio.

Despite this problem, extensive testing has shown that for contact systems for which $q$ is uncertain, the inclination, secondary temperature, and configuration are well determined. We also find that the choice of limb darkening coefficients has little effect on the final configuration. This effect is best illustrated in a (mass ratio, surface potential)-graph (Figure 1). The solutions for a given system are roughly parallel to the inner contact boundary. Our experience with approximately 15 systems using this approach (e.g., Leung 1977) indicates that if a system is contact, semidetached, or completely detached for one value of $q$, it will remain in the same configuration for all reasonable values of $q$.

\section{PHOTOMETRIC SOLUTIONS}

\section{a) AU Puppis}

The variability of AU Pup was discovered by O'Leary and O'Connell (1935). Chambliss (1968) obtained good photoelectric observations from Mount John Observatory with Johnson's $B$ and $V$ filters. He found that the system is very complex, and he attempted to derive a photometric solution with the Russell and Merrill (1952) approach. He reported the possibility of a third body (the third light), and discussed the uncertainty in the angle of external tangency and the rectification process. Chambliss obtained three sets of solutions by assuming three different ratios of radii $(0.7,0.8$, and 0.9$)$. He suggested that the primary eclipse is a transit, the secondary an occultation. He suggested that AU Pup might be a contact system since the sum of the side radii was 0.77 . Bossen (1972) attempted to improve on Chambliss's solution by including methods of perturbations and differential corrections. He concluded that the system was detached, with a sum of the radii of 0.57 . Bossen

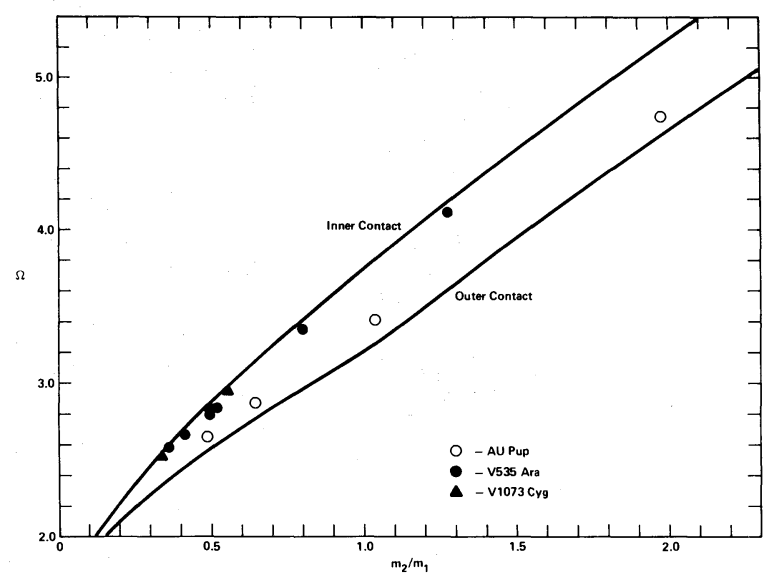

FIG. 1.-(Mass ratio, contact potential)-diagram. Circles, solutions for AU Pup; dots, solutions for V535 Ara; triangles, solutions for V1073 Cyg.

also assumed the primary to be a main-sequence star (A0 V) and calculated the absolute dimensions by assuming main-sequence bolometric magnitude and mass-luminosity relation. Unfortunately, the results obtained contradicted the assumptions. For example, he obtained $m_{1}=3 m_{\odot}$ and $R_{1}=2.7 R_{\odot}$. A mainsequence star of $3 m_{\odot}$ should have a radius of about $1.8 R_{\odot}$. We conclude that Bossen's analysis is questionable. There is no spectroscopic work reported for this system.

Chambliss's (1968) observations were employed in the analysis. In addition to the assumptions mentioned in $\S$ II, we further assumed that the polar effective temperature of component 1 was $T_{1}=9600 \mathrm{~K}$ (from spectral type AO and Morton and Adams 1968 temperature calibration); and the limb darkening coefficients, $x_{1}=x_{2}$, were equal to 0.48 and 0.56 for the yellow and blue wavelength regions, respectively (linear limb darkening coefficients derived from the grid model atmosphere of Carbon and Gingerich 1969 for $T=10,000 \mathrm{~K}$ and $\log g=4.0$ ). Chambliss assumed 0.6 and 0.6 for the corresponding values.

Unfortunately, the spectroscopic mass ratio is not known for AU Pup. We searched for solutions with different assumed values of $q$. The best solution (i.e., with smallest sum of residuals) is at mass ratio $q=$ 0.644 , while the solutions at $q=0.488,1.004$, and 1.969 give larger residuals (largest at 1.969). These solutions are shown in a (mass ratio, surface potential)diagram, Figure 1, as circles. Note that the solutions are roughly parallel to the inner-contact potential. This indicates that there may be uncertainty in the precise mass ratio, but the contact configuration is reliable. The solution of $q$ of 0.644 is adopted, and its photometric parameters are listed in Table 1. The theoretical light curves according to this solution together with the observed light curves are shown in Figure 2. The theoretical light curves (not shown) for mass ratios of 1.004 and 1.969 gave similar light curves except that they were shallower (i.e., brighter) at the primary minimum by about 0.005 and $0.010 \mathrm{mag}$, respectively. 
TABLE 1

Photometric Parameters for AU Puppis, V535 Arae, and V1073 Cygni

\begin{tabular}{|c|c|c|c|}
\hline Parameter & AU Puppis & V535 Arae & V1073 Cygni \\
\hline$L_{1} /\left(L_{1}+L_{2}\right)$ (yellow) $\ldots$ & $0.6349 \pm 0.0028$ & $0.7326 \pm 0.0044$ & $0.7700 \pm 0.0021$ \\
\hline$L_{1} /\left(L_{1}+L_{2}\right)$ (blue) $\ldots \ldots$ & $0.6435 \pm 0.0031$ & $0.7351 \pm 0.0051$ & $0.7740 \pm 0.0024$ \\
\hline$x_{1}=x_{2}($ yellow $\ldots \ldots \ldots$ & $0.48^{*}$ & $0.5^{*}-$ & $0.5^{*}-0.002$ \\
\hline$x_{1}=x_{2}$ (blue) $\ldots \ldots \ldots$ & $0.56^{*}$ & $0.6^{*}$ & $0.6^{*}$ \\
\hline$i$ (degrees) $\ldots \ldots \ldots \ldots \ldots$ & \pm 0.17 & $82.14 \pm 0.38$ & $68.30 \pm 0.19$ \\
\hline$\Omega_{1}=\Omega_{2} \ldots \ldots \ldots \ldots$ & $2.876 \pm 0.012$ & $2.5919 \pm 0.0072$ & $2.5382 \pm 0.0031$ \\
\hline$g_{1}=g_{2} \ldots \ldots \ldots \ldots \ldots$ & $1.0 \dagger$ & $1.0 \dagger$ & $1.0 \dagger$ \\
\hline$A_{1}=A_{2} \ldots \ldots \ldots \ldots$ & $1.0 \dagger$ & $1.0 \dagger$ & $1.0 \dagger$ \\
\hline$T_{1}(\mathrm{~K}) \ldots \ldots \ldots$ & $9600 \ddagger$ & $8750 \S$ & $8750 \S$ \\
\hline$T_{2}(\mathrm{~K}) \ldots \ldots \ldots \ldots \ldots \ldots$ & $9067 \quad \pm 16$ & $8572 \quad \pm 14$ & $8207 \quad \pm 21$ \\
\hline$m_{2} / m_{1} \ldots \ldots \ldots \ldots \ldots$ & $0.6443 \pm 0.0067$ & $0.3612 \pm 0.0037$ & $0.3400 \|$ \\
\hline$r_{1}$ (pole)... & $0.4372 \pm 0.0022$ & $0.4420 \pm 0.0014$ & $0.4487^{\prime \prime} \pm 0.0006$ \\
\hline$r_{1}($ side $) \ldots$ & $0.4729 \pm 0.0030$ & $0.4726 \pm 0.0018$ & $0.4809 \pm 0.0008$ \\
\hline$r_{1}(\mathrm{back})$ & $0.5313 \pm 0.0051$ & $0.4997 \pm 0.0023$ & $0.5083 \pm 0.0010$ \\
\hline$r_{2}($ pole $) ..$ & $0.3659 \pm 0.0023$ & $0.2759 \pm 0.0016$ & $0.2733 \pm 0.0006$ \\
\hline$r_{2}($ side $) ..$ & $0.3916 \pm 0.0030$ & $0.2874 \pm 0.0017$ & $0.2848 \pm 0.0008$ \\
\hline$r_{2}$ (back) $\ldots \ldots \ldots \ldots$ & $0.4720 \pm 0.0075$ & $0.3211 \pm 0.0027$ & $0.3200 \pm 0.0013$ \\
\hline$\%$ overcontact. ..... & $71.6--10$ & $2.5--1$ & $7.1--$ \\
\hline$\Omega_{\text {Inner }} \ldots \ldots \ldots \ldots \ldots$ & 3.143 & 2.574 & 2.553 \\
\hline$\Omega_{\text {outer }} \ldots \ldots \ldots \ldots$ & 2.770 & 2.375 & 2.343 \\
\hline Period. & 1.126418 & 0.629298 & 0.7858597 \\
\hline Spectral type.......... & A0 & A3 & A3 \\
\hline
\end{tabular}

* Linear limb darkening coefficients derived from the grid model atmospheres of Carbon and Gingerich 1969 for $T=9600 \mathrm{~K}$ and $\log g=4$.

$\dagger$ Assumed value.

$\ddagger$ Assumed temperature from spectral type and the Morton and Adams (1968) temperature calibration.

$\S$ Linear limb darkening coefficient derived from Carbon and Gingerich for $T=8750 \mathrm{~K}$ and $\log$ $g=4.0$.

|| Spectroscopic (FitzGerald 1965).

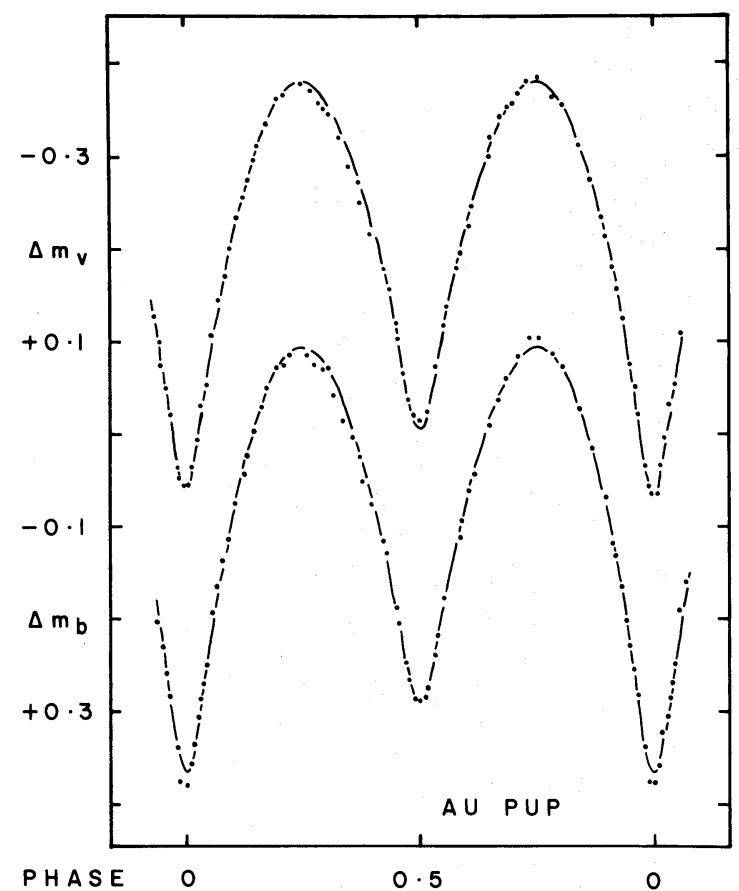

Fig. 2.-Light curves of AU Pup. Points, normal points of Chambliss (1968). The magnitudes are in the sense of AU Pup minus the comparison star. Solid lines, the theoretical light curves based on the parameters in Table 1.
In Figure 1, the observed light curves show a small asymmetry where the secondary maximum is slightly brighter. The agreement between the theoretical and the observed light curves is good except for a very small deficiency in light at around 0.35 phase. (A similar deficiency occurred for V535 Ara; see next section.) The configuration of AU Pup at 0.25 phase is shown in Figure 3. The primary minimum is a transit, which agrees with the suggestion of Chambliss (1968). The percentage of overcontact is very large, $72 \%$. The other systems known to have such a high

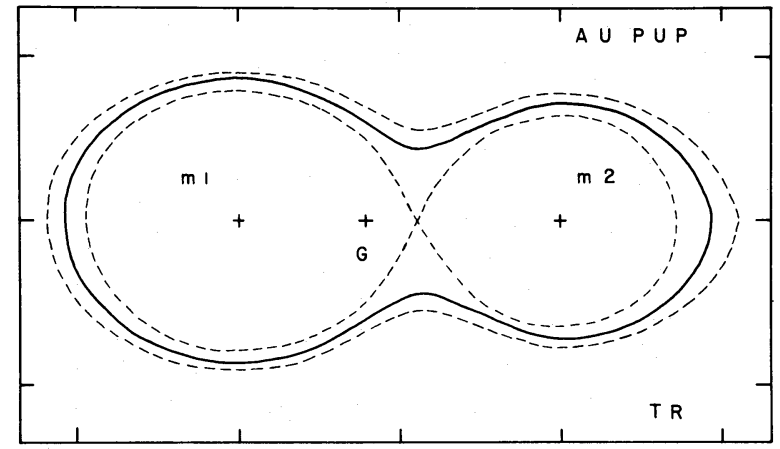

FIG. 3.- The contact configuration of AU Pup at 0.25 phase. Broken lines, the potential surfaces of inner and outer contacts. Plus signs, the centers of the components as well as the center of gravity. 
degree of overcontact are: SV Cen, $82 \%$ (Wilson and Starr 1976), and V701 Sco, 51\% (Wilson and Leung 1977).

There is general agreement in comparing our results with one of the solutions of Chambliss (1968, Table 3; ratio of radii, 0.80$): i, 81^{\circ}$ versus $78^{\circ} ; k$ (ratio of radii), 0.83 (side) versus 0.8 (assumed); and luminosity ratio, 0.57 versus 0.74 , respectively. Chambliss found that $9 \%$ of the light of the system cannot be accounted for in his solutions and suggested "extra-stellar material lying between, but not enveloping the two stars." We find that there is no need to invoke an extra source, although Wilson and Devinney's approach does allow one to use a third light as an adjustable parameter.

\section{b) V535 Arae}

V535 Ara = BV 419 was discovered by Ströhmeier (1964). Chambliss (1967) observed this system photoelectrically from Mount John Observatory using Johnson's $B$ and $V$ filters. No change in period was detected from his study of times of minimum. He obtained a photometric solution by employing the Russell and Merrill approach. He concluded that the primary minimum was a transit and the secondary minimum was an occultation, and the eclipses were complete. He reported the ratio of radii to be 0.54 and the sum of radii to be 0.75 . Spear (1974) reported Strömgren photometry at secondary maximum and phases 0.25 and 0.75 (out of eclipse). There is no spectroscopic work reported on the system.

Chambliss's (1967) observations were employed in this study. We assumed a polar effective temperature of component $1, T_{1}=8570 \mathrm{~K}$ (from spectral type A3), and limb darkening coefficients, $x_{1}=x_{2}$, equal to 0.5 and 0.6 for yellow and blue, respectively (from Carbon and Gingerich 1969, for $T=8500 \mathrm{~K}$ and $\log g=4.0$ ). Chambliss assumed 0.4 and 0.4 for the corresponding values.

As in the situation for AU Pup, the spectroscopic mass ratio for V535 Ara is not known. Again, several values of $q$ were tried. These solutions are shown in Figure 1 as dots. Note that they are roughly parallel to the inner contact potential, with the smallest mass ratio almost touching the line. The solution for a mass ratio of 0.3612 gives a significantly smaller sum of residuals. Thus, this solution is adopted for our study, and its photometric parameters are listed in Table 1.

The theoretical, as well as the observed light curves, are shown in Figure 4. The agreement between them is good, except for a small deficiency in light around 0.32 phase. A similar deficiency was also detected in AU Pup. The configuration at 0.25 phase is shown in Figure 5. The primary minimum is a transit which agrees with Chambliss's suggestion (1967). The degree of overcontact is $2.5 \%$, indicating marginal contact only.

A comparison of our solution with that of Chambliss is as follows: $i, 82^{\circ}$ versus $78^{\circ} ; k, 0.58$ (side) versus 0.54 ; and luminosity ratio 0.73 (yellow) versus 0.71 , respectively. Our solution shows that the eclipse at

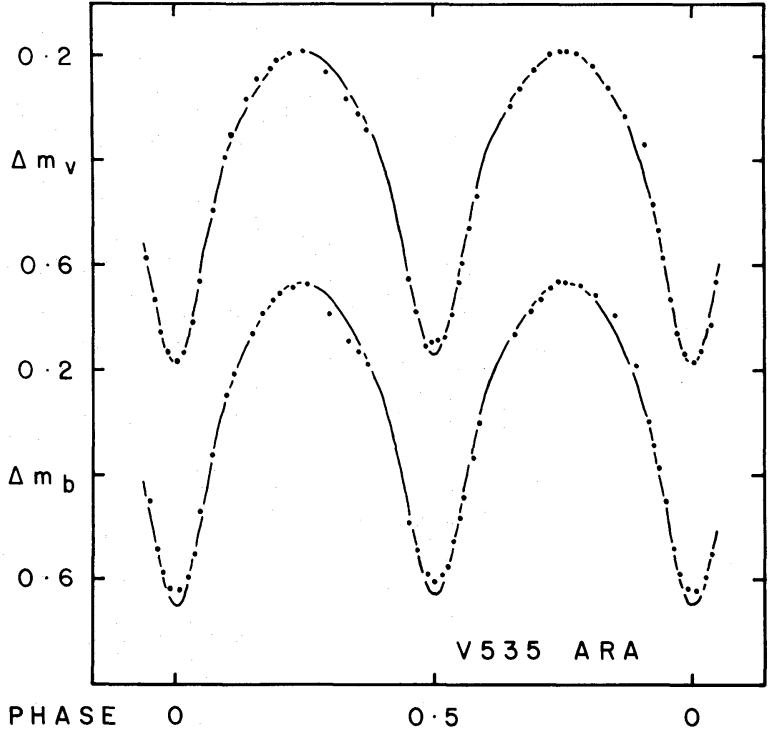

FIG. 4.-Light curves of V535 Ara. Points, normal points of Chambliss (1967). The magnitudes are in the sense of V535 Ara minus the comparison star. Solid lines, the theoretical light curves based on the parameters in Table 1 .

the secondary minimum is complete (from 0.49 to 0.51 phases) in agreement with Chambliss's suggestion.

\section{c) V1073 Cygni}

V1073 Cyg = BV 342 was discovered by Ströhmeier (1960). FitzGerald (1965) observed the system spectroscopically and derived an orbital solution for this double-lined spectroscopic binary. He also reported an elliptical orbit with $e=0.115$. Kondo (1966) obtained photoelectric observations of this system from Flower and Cook Observatory using Johnson's $B$ and $V$ filters. He derived a photometric solution by employing the Russell and Merrill approach. He also obtained the absolute dimensions of this system by combining his solution with that of FitzGerald (1965).

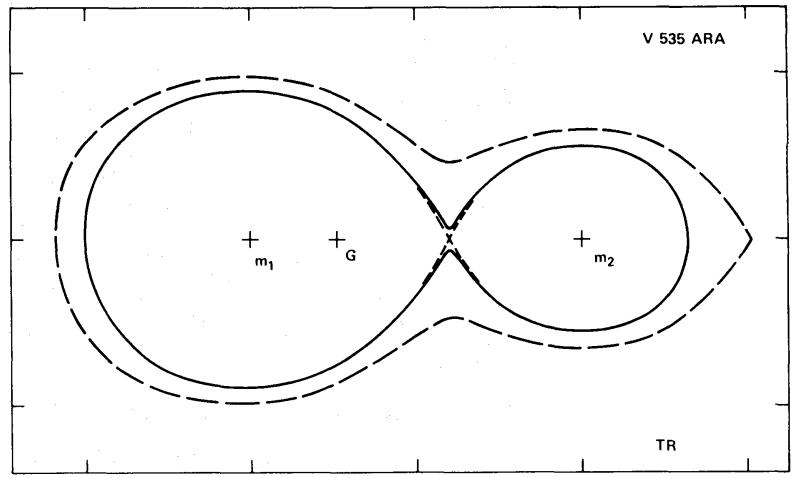

FIG. 5.-The contact configuration of V535 Ara at 0.25 phase. Broken lines, the potential surfaces of inner and outer contacts. Plus signs, the centers of the components as well as the center of gravity. 
He concluded that V1073 Cyg may be a contact system. Bendinelli et al. (1967) observed photoelectrically in yellow and blue filters, and analyzed their observations in similar manner to that of Kondo obtaining similar results. Kruseman (1968) observed photoelectrically in 1962, 1964, and 1965 and reported a possible change in the light curve over the years.

Kondo's observations (1966) were employed for the analysis. We assumed a polar effective temperature of component $1, T_{1}=8500 \mathrm{~K}$ (from spectral type A3, the same as V535 Ara); limb darkening coefficients the same as V535 Ara, i.e., 0.5 and 0.6 for yellow and blue, respectively. The spectroscopic mass ratio of 0.34 by FitzGerald (1965) was adopted. A few different values of mass ratio were attempted, but the spectroscopic value gave the best solution (i.e., smallest sum of squared residuals). The different solutions are shown in Figure 1 as triangles. They are very close to the inner surface potentials.

The photometric solution of V1073 Cyg is also listed in Table 1. The theoretical and observed light curves are shown in Figure 6. There is general agreement between them except for the descending branch of the secondary minimum. The theoretical light curves in these intervals are slightly brighter. It seems that all three of these intermediate-mass spectral type A contact systems show some complications in this descending branch. The configuration at phase 0.25 is shown in Figure 7. The primary minimum is a transit in agreement with Kondo (1966). The degree of overcontact is small, $7 \%$. Kondo suggested a contact configuration from his study of absolute dimensions of this system.

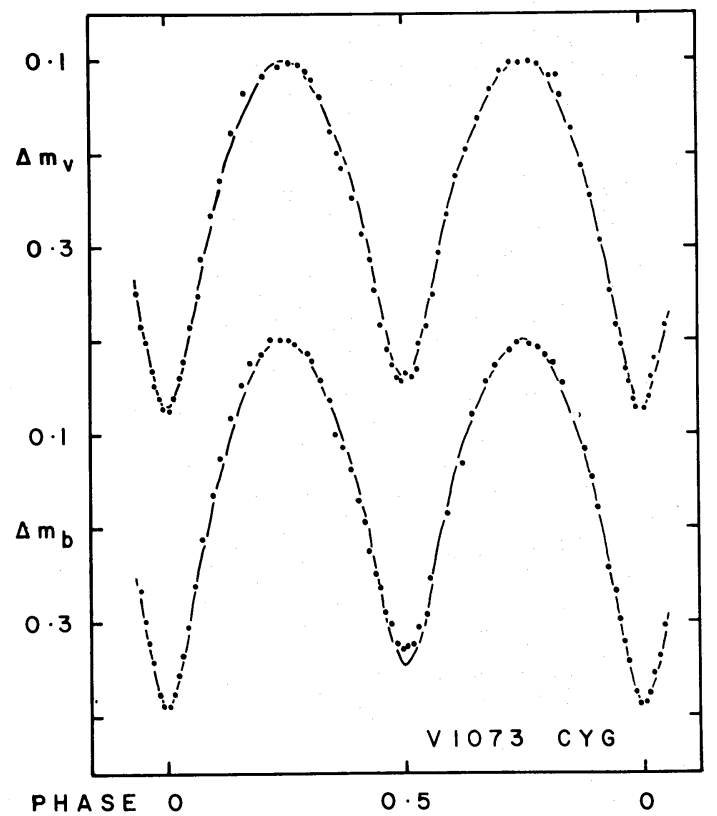

FIG. 6.-Light curves of V1073 Cyg. Points, normal points of Kondo (1966). The magnitudes are in the sense of V1073 Cyg minus the comparison star. Solid lines, the theoretical light curves based on the parameters in Table 1.

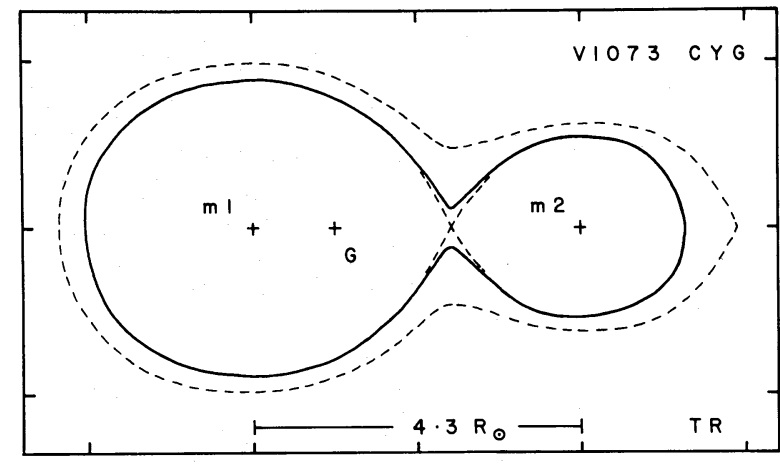

FIG. 7.-The contact configuration of V1073 Cyg at 0.25 phase. Broken lines, the potential surfaces of inner and outer contacts. Plus signs, centers of the components as well as the center of gravity.

A comparison of our results with that of Kondo's are as follows: $i, 68^{\circ}$ versus $65^{\circ} ; k, 0.59$ (side) versus 0.61 ; and luminosity ratio 0.30 versus 0.58 .

FitzGerald (1965) reported an eccentricity of $e=0.115$, which is large. Our solution gives a contact configuration which implies that $e=0$. If one were to assume that the system is detached, the separation would have to be very small. Thus, the tidal distortion of the component would remain very large. Under this situation, the tidal force would make the elliptical orbit circular rather quickly. Therefore, we conclude that it is rather unlikely that V1073 Cyg has an elliptical orbit. One may suspect that the line profiles could be seriously affected by the distortion of the components. Thus, the radial velocity curves may not be truly reflecting the orbital velocities of the mass center of the components. Under a contact configuration (also true for near contact), the reflection effect, tidal distortion and eclipses have significant effect on the observed radial velocity.

In calculating the absolute dimensions of the system, we adopted $e=0$, a mass ratio of 0.34 , and $K_{1}=242 \mathrm{~km} \mathrm{~s}^{-1}$ from FitzGerald (1965). The result is listed in Table 2. There is general agreement with Kondo (1966, Table 6). With the adopted $K_{1}$, period, and the parameters in Table 1, we computed the radial velocity of V1073 Cyg. In our computation we included the following effects: effects of reflection, tidal distortion, and eclipses (see Wilson and Sofia 1976 for the detailed technique and their subroutine). The computed radial velocity curves together with the observations of FitzGerald (1965) are shown in Figure 8 . Note that the computed radial velocity

TABLE 2

Absolute Dimensions of V1073 Cygni

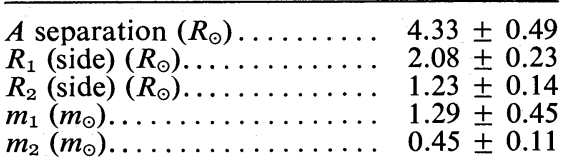

Note.--Radius for a $1.3 m_{\odot}$ ZAMS star, $1.1 R_{\odot}$. 


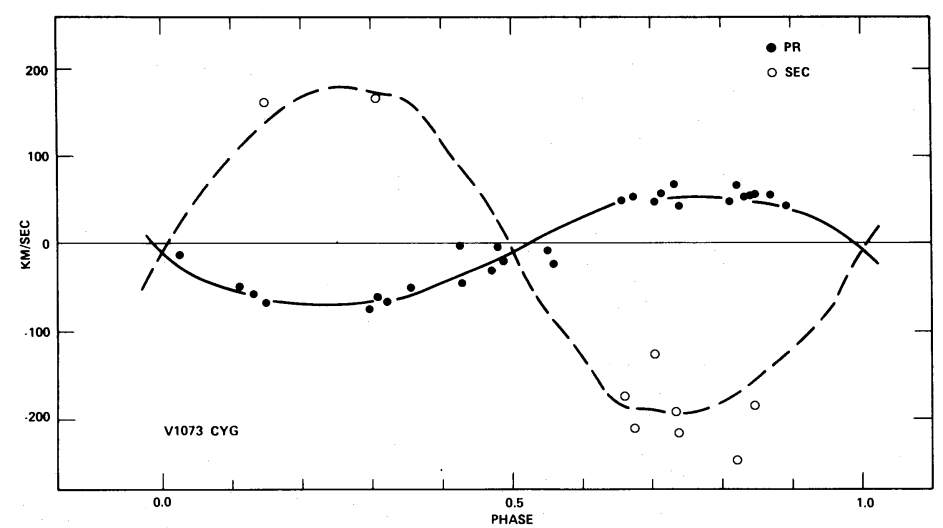

FIG. 8.-Radial velocity curves of V1073 Cyg. Dots, observations of FitzGerald (1965). The smooth curves are the computed radial velocity curves based on $K_{1}=242 \mathrm{~km} \mathrm{~s}^{-1}, V_{0}=-8.1 \mathrm{~km} \mathrm{~s}^{-1}, e=0$, and the photometric solution listed in Table 1 .

curves are not symmetric about phases 0.25 and 0.75 , even though we assumed an eccentricity $e=0$. This is because the corrections for the reflection effect, tidal distortion, and eclipses are significant, from -12 to $+5 \mathrm{~km} \mathrm{~s}^{-1}$, and from -6 to $+16 \mathrm{~km} \mathrm{~s}^{-1}$ for the primary and the secondary, respectively (see Table 3 ). There is good agreement between the computed and the observed radial velocity without invoking an elliptical orbit. Therefore, we conclude that the eccentricity derived from spectroscopic work is not real.

\section{EVOLVED CONTACT SYSTEMS}

How can we tell if a contact system is zero-age or evolved? We can settle this question if we know the ages and masses, or the masses and radii, or if the system happens to be a member of a star cluster. We are interested in gaining some insight into this question when we know only the period and the spectral type of the system.

The period of a binary is related to the sum of the masses and their separation through Kepler's law.

TABLE 3

Radial Velocity CORRection of V1073 CYGN *

\begin{tabular}{|c|c|c|}
\hline Phase & $\Delta \mathrm{RV}_{1}$ & $\Delta \mathrm{RV}_{2}$ \\
\hline 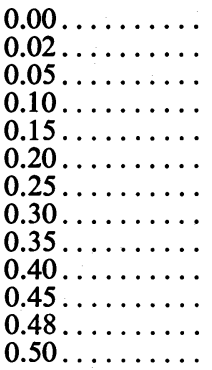 & $\begin{array}{r}0 \\
-7.1 \\
-11.7 \\
-8.2 \\
-3.4 \\
-1.4 \\
+4.3 \\
+4.7 \\
+4.5 \\
+2.1 \\
+1.0 \\
+0.4 \\
0\end{array}$ & $\begin{array}{c}0 \\
-0.9 \\
-2.1 \\
-3.8 \\
-5.4 \\
-6.3 \\
-5.6 \\
-3.0 \\
+2.3 \\
+7.5 \\
+16.0 \\
+10.4 \\
0\end{array}$ \\
\hline
\end{tabular}

* Assuming $i=68^{\circ} 3$, mass ratio $=$ 0.343 , and $K_{1}=65.9 \mathrm{~km} \mathrm{~s}^{-1}$ (including effects of reflection, tidal distortion, and eclipses).
Let us define the critical period as corresponding to the time when both components are just filling their inner Roche surfaces for a given mass ratio. Under this condition, the fractional side-radius of the primary (star 1 , in our case), $r_{\mathrm{pr}}$, is equal to 0.375 and 0.442 for the mass ratio, $q=m_{2} / m_{1}, 1.0$ and 0.5 , respectively. The separation $A$ (in $R_{\odot}$ ) can be expressed by the following equation:

$$
A=\frac{R_{\mathrm{pr}}(\text { side })}{r_{\mathrm{pr}}(\text { side })}, \quad R_{\mathrm{pr}} \text { in } R_{\odot} .
$$

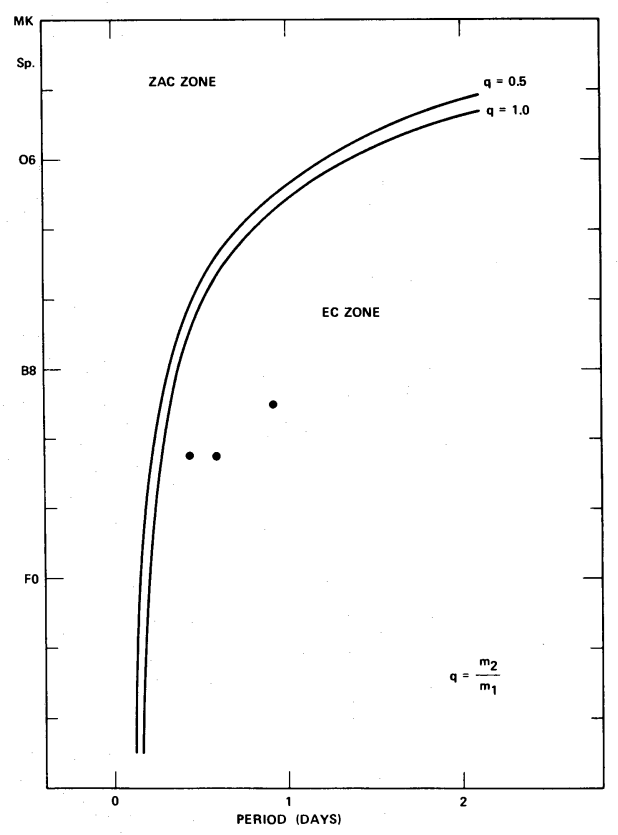

Fig. 9.-The solid lines in this period-spectral-type diagram are the locations of a zero age system just in contact. One is for a mass ratio of 1.0 , the other for 0.5 , assuming the ZAMS mass-radius relation of Stothers (1972). The dots represent the contact systems V535 Ara, V1073 Cyg, and AU Pup (from left to right). ZAC stands for zero-age contact, and EC stands for evolved contact. 
Then, the critical contact period can be expressed as follows:

$$
P=\frac{1}{\left[74.4(1+q) r_{\mathrm{pr}}\right]^{1 / 2}} \frac{R_{\mathrm{pr}}^{3 / 2}}{m_{\mathrm{pr}}^{1 / 2}} \text { days }
$$

where $m_{\mathrm{pr}}$ in $m_{\odot}, R_{\mathrm{pr}}$ in $R_{\odot}$. In general, the side radius is very close to the mean radius of the star. Thus, the critical period for zero-age contact can be calculated by adopting the zero-age main-sequence (ZAMS) mass-radius relation. Theoretical zero-age contact periods are calculated for $q=1.0$ and 0.5 from the ZAMS mass-radius relation of Stothers (1972) ( $X_{e}=0.739, Z_{e}=0.044$, Cox-Stewart opacity). Generally, the masses of a binary system are not known, whereas the spectral type may be readily available. Thus, we convert the theoretical zero-age (contact period, mass)-relation into a (period, spectral type [MK])-relation. The result is shown in Figure 9. These critical contact boundaries divide the contact systems into zero-age contact systems on the left and evolved contact systems on the right in the (period, spectral type)-plane. The farther a system falls to the left of the boundaries, the greater the degree of overcontact. On the other hand, the farther to the right of the boundaries the system is located, the more evolved the system is. The distance does not necessarily indicate the degree of overcontact. However, caution should be taken in applying this (period, spectral type)relation. The period of the system is usually very accurate whereas the spectral type is more uncertain, especially when a system is in contact. In some cases an error of two subtypes would move the system in or out of the boundaries!

V1073 Cyg, having radii larger than the corresponding values for the ZAMS stars ( $\$$ III $c$ ), falls in the evolved contact (EC) zone in Figure 9 as predicted. Both AU Pup and V535 Ara also fall in the EC region, which suggests that these systems are evolved contact systems.

It is not possible to tell whether a given evolved contact system originated from a detached system or from a zero-age contact system. This is a very involved problem and beyond the scope of this paper.

We thank Dr. Yoji Kondo for sending us the normal points of V1073 Cyg. We are indebted to Dr. Robert E. Wilson for his radial velocity subroutine. D. P. S. would like to acknowledge support from the following scholarships during the course of his study at the University of Nebraska: Westinghouse Scholarship, National Merit Scholarship, Regents Scholarship, Harkson Scholarship, and Stebbins Scholarship; and at Caltech: Earle C. Anthony Fellowship. K. C. L. would like to acknowledge the support from the University of Nebraska Research Grants. He also thanks Dr. G. K. Oertel for his constant encouragement. We are grateful to Dr. F. B. Wood for sending information from the University of Florida Eclipsing Binary Card Catalog.

\section{REFERENCES}

Bendinelli, O., Delli Ponti, C., Catalano, S., and Cristaldi, S. 1967, Mem. Soc. Astr. Italiana, 38, 763.

Bossen, H. 1972, Astr. Ap., 17, 362.

Carbon, D. F., and Gingerich, O. 1969, in Theory and Observations of Normal Stellar Atmospheres, ed. O. Gingerich (Cambridge: MIT Press), p. 377.

Chambliss, R. C. 1967, A.J., 72, 512 .

- 1968, A.J., 73, 1024.

Eggen, O. J. 1961, Royal Obs. Bull, No. 31, E101.

FitzGerald, P. 1965, Pub. David Dunlap Obs., 2, 417.

Hill, G., and Hutchings, J. B. 1970, Ap. J., 162, 265.

Kondo, Y. 1966, A.J., 71, 54.

Kopal, Z. 1959, Close Binary Systems (New York: Wiley). Kruseman, P. 1968, Bull. Astr. Inst. Netherlands Suppl., 2, 377. Leung, K. C. 1977, IAU Trans. XVI B, Report of Commission 42 (Dordrecht: D. Reidel), p. 283.

Leung, K. C., and Schneider, D. P. 1977, Ap. J., 211, 844.

Leung, K. C., and Wilson, R. E. 1977, Ap. J., $211,853$.

Lucy, L. 1968, Ap. J., 151, 1123.

Mochnachi, S. W., and Doughty, N. A. 1972, M.N.R.A.S., 156, 243.

Morton, D. C., and Adams, T. F. 1968, Ap. J., 151, 611.

O'Leary, W., and O'Connell, D. 1935, Astr. Nach., 257, 391.

Russell, H. N., and Merrill, J. E. 1952, Contr. Princeton Univ. Obs., No. 26.

Spear, G. G. 1974, Bull. AAS, 6, 222.

Stothers, R. 1972, Ap. J., 175, 432.

Ströhmeier, W. 1960, IAU Circ., No. 1735.

. 1964, Inf. Bull. Var. Stars, No. 49.

Wilson, R. E., and Devinney, E. J. 1971, Ap. J., 166, 605.

Wilson, R. E., and Leung, K. C. 1977, Astr. Ap., 61, 137.

Wilson, R. E., and Sofia, S. 1976, Ap. J., 203, 182.

Wilson, R. E., and Starr, T. C. 1976, M.N.R.A.S., 176, 625.

K. C. LEUNG: 309-RB, Office of Energy Research, U.S. Department of Energy, Washington, DC 20545

D. P. SCHNEIDER: Department of Astronomy, 105-24, California Institute of Technology, Pasadena, CA 91125 